Review

\title{
Current Status and Perspective of Immunotherapy in Gastrointestinal Cancers
}

\author{
Jung Hoon Kim, Bum Jun Kim, Hyeong Su Kim, Jung Han Kim $\bowtie$ \\ Division of Hemato-Oncology, Department of Internal Medicine, Hallym University Medical Center, Hallym University College of Medicine, Chuncheon, South \\ Korea. \\ $\triangle$ Corresponding author: Jung Han Kim, MD, PhD, Department of Internal Medicine, Kangnam Sacred-Heart Hospital, Hallym University Medical Center, \\ Shingilro-1, Youngdeungpo-Gu, Seoul 07441, South Korea. harricil@hotmail.com, harricil@hallym.or.kr Telephone: +82-2-8345414 Fax: +82-2-8464669. \\ (C) Ivyspring International Publisher. Reproduction is permitted for personal, noncommercial use, provided that the article is in whole, unmodified, and properly cited. See \\ http://ivyspring.com/terms for terms and conditions.
}

Received: 2016.05.18; Accepted: 2016.07.09; Published: 2016.07.18

\begin{abstract}
Cancer immunotherapy is at dawn of the Renaissance after the Medieval Dark Ages. Recent advances of understanding tumor immunology and molecular drug development are leading us to the epoch of cancer immunotherapy. Some types of immunotherapy have shown to provide survival benefit for patients with solid tumors such as malignant melanoma, renal cell carcinoma, or non-small cell lung cancer. Several studies have suggested that immune checkpoint inhibition might be effective in some patients with gastrointestinal cancers. However, the era of cancer immunotherapy in gastrointestinal cancers is still in an inchoate stage. Here we briefly review the current status and perspective of immunotherapeutic approaches in patients with gastrointestinal cancers.
\end{abstract}

Key words: Gastrointestinal cancers; Immunotherapy; Cancer vaccines; Immune checkpoint inhibitors; Review.

\section{Introduction}

Gastrointestinal cancers are still a leading cause of death worldwide. Gastric cancer (GC) and colorectal cancer (CRC) have ranked third and fourth among the most common causes of cancer death, despite advances of surgical resection, chemotherapy, and molecular targeted therapy [1,2]. Although application of molecular targeted agents such as cetuximab, bevacizumab, trastuzumab, and ramucirumab led to meaningful improvement in the prognosis of patients with gastrointestinal cancer, overall survival outcomes remain poor. Thus, gastrointestinal cancers still need a novel approaches. The recent expansion of our understanding of anti-tumor immunity and increasing clinical experience of new cancer immunotherapy have raised hope that we can treat cancer effectively with immunotherapeutic approaches.

The category of cancer immunotherapy includes broad strategies to utilize the immune system of patients to fight cancer. Early approaches usually transfused certain types of immune cells or cytokines, such as cytotoxic T lymphocytes [3], high dose interleukin (IL)-2 [4], or interferon (IFN)- $\mathrm{a}$ [5], directly into patients. Most studies failed to elicit meaningful response, which was probably attributed to immunosuppressive tumor environment or heavy toxicity induced by activated autoimmune reaction [3-5]. However, recent advances of understanding tumor immunology and molecular drug development are leading us to the epoch of cancer immunotherapy.

Immunotherapy targeting cancer has been assumed to be beneficial mainly in tumors with high immunogenicity by nature $[4,6]$. However, some approaches to circumvent immunosuppression including programmed death-1 (PD-1)/programmed death ligand-1 (PD-L1) blockade were successful to achieve significant responses in cancers which hardly retain immunogenic nature [7]. Clinical trials of anticancer immunotherapy are being expanded to various types of cancer. Accordingly, clinical oncologists treating patients with gastrointestinal cancers should also consider immunotherapy as a 
therapeutic option.

Here we briefly review the current status and perspective of various immunotherapeutic approaches including adoptive cellular therapy, cancer vaccines, and immune checkpoint inhibitors, mainly in patients with gastrointestinal cancers.

\section{Adoptive cellular therapy}

Adoptive cellular therapy is to collect $\mathrm{T}$ cells from cancer patients and cultivate them in the laboratory and give back to the patients for anticancer effects. Its rationale is based on the extensive studies showing that tumor-infiltrating $\mathrm{T}$ lymphocytes were a better prognostic factor for survival [8]. Unfortunately, none of adoptive cellular therapy has been approved for cancer patients. The major obstacle interfering with adoptive cellular therapy is an immunosuppressive environment surrounding tumor. As we can see from the recent PD-1/PD-L1 studies, moreover, immunosuppressive mechanisms in cancer patients vary according to individuals. To elicit anti-tumor effects, therefore, this type of treatment should overcome the intrinsic immunosuppressive mechanisms surrounding tumors.

Currently, improved culture technology and more detailed approach are in use to inspire tumor specificity of $\mathrm{T}$ cells and surmount immune escape mechanisms. In one trial, CD4+ T helper 1 (Th1) cells acquired from sentinel lymph nodes were clonally expanded and administered to CRC patients [9]. The results were encouraging to induce complete tumor regression in four out of nine patients with stage IV CRC. However, the complicated process to collect sentinel lymph nodes surgically and yield efficient $\mathrm{T}$ cells remains as a major hurdle. Cytokines can be used as a tool to separate effective T cells from the blood of cancer patients. Jiang et al. isolated cytokine-induced killer (CIK) cells from their peripheral blood mononuclear cells of patients with advanced GC and cultured them with IL-1a and anti-CD3 monoclonal antibody $(\mathrm{mAb})$ [10]. The CIK cells were transfused into patients in combination with oxaliplatin and 5-fluorouracil/leucovorin (FOLFOX) chemotherapy. The patients treated with CIK cells and FOLFOX chemotherapy showed higher overall response rate, compared to those treated with FOLFOX alone (56.3\% vs. $48 \%, P<0.05)$.

\section{Cancer vaccines}

Vaccines as a tool for treating cancer have been studied to augment and maintain anticancer immune response in patients with cancer. Cancer vaccines have been tried preferentially in highly immunogenic type of cancers, such as renal clear cell carcinoma
(RCC) [11], malignant melanoma [12], or non-small cell lung cancer (NSCLC) [13]. Peptide, protein, whole tumor cells or dendritic cell (DC)-based vaccines have been also tried in gastrointestinal cancers [14]. Peptide vaccines are the simplest form of anticancer vaccination. Early trials of peptide vaccination for CRC targeted carcinoembryonic antigen (CEA) and mainly aimed to manipulate immune activity of patients. However, clinical trials failed to draw significant CEA-specific antibody responses [15]. Vaccination with autologous tumor-derived heat shock protein peptide complex Gp 96 showed encouraging results in some patients with metastatic CRC: disease-free survival and overall survival (OS) at 2 years of immune responding patients were prolonged, compared to those of non-responding patients $(51 \%$ vs. $8 \%, P=0.0001,100 \%$ vs. $50 \%, P=$ 0.001) [16].

DCs are the most potent antigen-presenting cells which activate $\mathrm{T}$ cells and express immune stimulatory molecules. To enhance antitumor immune response, tumor lysate-pulsed DCs have been tried in patients with various cancers including malignant melanoma, CRC, NSCLC, prostate cancer, RCC [11], and brain tumor [17]. The positive results with survival benefit were reported by a phase III IMPACT trial with sipuleucel-T, an autologous DC therapeutic vaccine designed to enhance the immune $\mathrm{T}$ cell response to prostatic acid phosphatase in patients with metastatic castration-resistant prostate cancer [18]. In patients with GC, however, DCs pulsed with Her-2/neuropeptides and DCs pulsed with MAGE-3 peptides have been tried to show no significant effect $[19,20]$.

DC as a therapeutic option to defeat cancer has some shortcomings because it has a short life and its immune activity mainly depends on cellular maturity. Some researchers adopted IFN- $\gamma$ and lipopolysaccharide (LPS) to develop high IL-12p70-producing DCs. However, DCs matured with IFN- $\gamma$ and LPS were problematic because of the narrow temporal window for IL-12p70 production [21]. Kim et al. produced DCs in which the PI3K/AKT pathway was activated by downregulating phosphatase and tensin homolog with small interfering RNA (siRNA) and observed increased survival of DCs from the apoptotic death in mice [22]. Preventive removal of regulatory $\mathrm{T}$ cells may be helpful to promote tumor-directed T-cell response in DC vaccination [23]. Morse et al. reported promising clinical data of DCs for gastrointestinal cancer [24]. CRC patients whose metastatic lesion had been resected were immunized with autologous DCs modified with poxyvectors encoding CEA and Mucin-1 (MUC1) or with the same poxyvectors plus 
granulocyte-macrophage colony stimulating factor (GM-CSF). Relapse-free survival (RFS) at 2 years showed no significant difference between two immunized groups ( $47 \%$ vs. $55 \%$, respectively), but as one group, OS of vaccinated patients were significantly better than those of contemporary, unvaccinated control patients (not reached vs. 44.1 months, respectively, $P<0.0001$ ). A randomized trial conducted by Barth et al. vaccinated patients with resected metastatic CRC with DCs which were either activated or not activated by CD40L ex vivo [25]. There was also a significant difference of RFS between responders and non-responders $(63 \%$ vs. $18 \%, P=$ 0.037). Recently, clinical outcomes of DC vaccination combined with CIK therapy in hepatobiliary and pancreatic cancer were reported [26]. Median OS of the immunotherapy group was significantly longer than that of the non-immunotherapy group (134 days vs. 115 days, $P=0.014)$. Sixty-one percent of patients who received immunotherapy experienced a positive, delayed-type hypersensitivity response, but adverse effects were generally manageable.

Cancer vaccines have not been validated in patients with gastrointestinal cancers. However, this type of treatment holds the potential to produce the persistent or even permanent anticancer effect in some patients. Different clinical outcomes between sipuleucel-T for prostate cancer and peptide vaccination targeting CEA for CRC suggest that it is crucial to define specific tumor antigens.

\section{Immune checkpoint inhibitors}

Immune checkpoint blockade is the most spotlighted cancer treatment at present. Current great attention on anticancer immunotherapy mainly depends on latest advance in this area. Immune checkpoint pathways have roles as inhibitors of $\mathrm{T}$ cell function by molecular signaling between $\mathrm{T}$ cells and antigen-presenting cells. The major approaches to harnessing anticancer immunity have come from the development of the inhibitory antibodies which block immune checkpoints, such as cytotoxic T-lymphocyte-associated antigen 4 (CTLA-4), PD-1, and PD-L1.

Ipilimumab is a mAb to block CTLA-4 receptor. Ipilimumab reinforced immune function of $\mathrm{T}$ cells against tumor and improved overall survival for patients with metastatic malignant melanoma [27]. Food and Drug Administration (FDA) approved ipilimumab as the first immunotherapeutic agent for patients with advanced malignant melanoma. In a pilot study, however, ipilimumab showed no response in three patients with CRC [28]. In addition, phase II trials with tremelimumab, a fully humanized anti-CTLA-4 mAb, drew meaningful response in neither GC nor CRC $[29,30]$.

Other promising targets are PD-1 and PD-L1 which are the members of the CD28 and B7 families. Communication between PD-1 expressed on activated CD8+ T cells and PD-L1 expressed on tumor cells is noted to suppress antitumor $\mathrm{T}$ cell immune activity [31]. PD-1/PD-L1 blocking treatment has been shown to enhance survival benefit for patients with metastatic melanoma [32] and NSCLC [33,34]. In 2015, FDA approved nivolumab, a PD-1 checkpoint inhibitor, for the treatment of advanced NSCLC, and pembrolizumab, another PD-1 inhibitor, for NSCLC patients with PD-L1 expression in their tumor cells.

Now investigators are trying to expand its indication to other type of tumors. Unlike CTLA-4 blockade, PD-1/PD-L1 pathway inhibitors seem to be promising because they achieved significant response in tumors which are regarded as not highly immunogenic. Inferring from the observation that immense toxicity by systemic autoimmune reaction rarely occurred, in addition, PD-1/PD-L1 blockade treatment appears to be more tumor-specific than CTLA-4 blockade [32,35]. Recently, several clinical trials have been conducted to evaluate the efficacy of PD-1 blockade in CRC. Llosa et al. demonstrated that defect in mismatch repair (MMR) resulting in microsatellite instability (MSI) is associated with high CD8+ cytotoxic T cell and Th1-type cell infiltration around the tumor [36]. Increased mutation burden in such tumor is responsible for immune reaction and patient with increased mutation burden may be a good candidate for immune checkpoint inhibitor. In 2015, Le DT et al. reported the efficacy of pembrolizumab in MMR-deficient CRC [37]. They conducted a phase II study to evaluate the activity of pembrolizumab in 41 patients with refractory metastatic cancer with or without MMR-deficiency: 11 patients had MMR-deficient CRC and 21 had MMR-proficient CRC. Pembrolizumab showed considerable efficacy with MMR-deficient CRC but didn't with MMR-proficient CRC. The immune-related objective response rate was $40 \%$ in MMR-deficient CRC and 0\% in MMR-proficient CRC and the progression-free survival (PFS) rate was 78\% in MMR-deficient CRC and 11\% in MMR-proficient CRC. In ECCO 2015, preliminary results from KEYNOTE-028 reported that overall response rate (ORR) and disease control rate of pembrolizumab in patients with PD-L1 positive advanced CRC were $4.3 \%$ and $21.7 \%$, respectively [38]. Only one responder was the sole patient who had microsatellite instability high (MSI-H) tumor. MSI-H tumors are more immunogenic [39] and have a significantly higher number of infiltrating FOXP3+ cells compared with microsatellite stable (MSS) tumors [40]. A high level of 
FOXP3+ cells was a favorable prognostic factor in advanced CRC $[41,42]$. These findings suggest that MSI status rather than PD-L1 status might be more promising predictive marker for immune checkpoint inhibitor in CRC.

In patients with GC, the results of PD-1/PD-1 blockade are mostly preliminary at present. In 2015 ASCO gastrointestinal symposium, Muro et al. presented the results of the KEYNOTE-012 trial that investigated the efficacy of pembrolizumab in patients with advanced GC whose tumors had more than $1 \%$ of PD-L1 expression. Among 39 patients enrolled, 22.1\% showed objective response, and median time to response was 8 weeks, with median duration of response of 24 weeks [43]. At 6 month, $24 \%$ of patients showed no signs of disease progression, and $69 \%$ remained alive. The median PFS and OS were 1.9 months and 11.4 months, respectively. The common adverse events included fatigue $(17.9 \%)$, decreased appetite (12.8\%), hypothyroidism (12.8\%), and arthralgia (10.3\%). Severe adverse events (grade 3 or 4 ) were observed in 5 patients $(12.8 \%)$, and one of these patients died for treatment-associated pneumonitis.

In 2016 ASCO gastrointestinal symposium, the preliminary results of the CheckMate-032 study of nivolumab in advanced and metastatic gastric or gastroesophageal junction cancer (GEC) were presented [44]. The CheckMate-032 trial is a two-phase study being conducted in patients with advanced solid tumors in the United States and Europe. The first part of the CheckMate-032 study focused on evaluating the efficacy of nivolumab in patients with advanced cancers that were previously treated. Eligible patients could enter the study without regard to PD-L1 expression levels in tumor samples. All patients received nivolumab $3 \mathrm{mg} / \mathrm{kg}$ intravenously every 2 weeks until progressive disease or intractable toxicity. Of the 59 patients with GC/GEC included in the phase I portion of the trial, $49(83 \%)$ had received at least 2 prior therapeutic regimens. In 40 patients, tissues samples were tested for PD-L1, and of these, 38\% harbored tumors categorized as PD-L1 positive (i.e., $\geq 1 \%$ membranous PD-L1 staining on tumor cells). The ORR was $14 \%$, with 1 complete response and 7 partial responses, and the median duration of response was 7.1 months. With 11 patients with stable disease, the disease control rate was $32 \%$. Of interest, the ORR in patients with PD-L1-positive and PD-L1-negative tumors was $27 \%$ and $12 \%$, respectively, suggesting that PD-L1 expression may be tied to higher response rates. The Median OS was 5.0 months and $36 \%$ of patients remained alive at 12 months.

In the mutational landscape study, molecular signature of smoking was predictive of durable clinical benefit of pembrolizumab [45]. Tumor tissue analytic study of KRAS-mutant lung cancer reported that PD-1 expression was more frequently observed in smokers and associated with more pack-years [46]. These results suggest that immune checkpoint inhibitors may show good response in some type of gastrointestinal cancers which have causative mutagens, such as upper esophageal carcinoma or C. sinensis-associated cholangiocarcinoma [47]. In ECCO 2015, pembrolizumab in heavily-pretreated patients with PD-L1 positive adenocarcinoma of the biliary tract or gallbladder reported the ORR of $17.4 \%$ and stable disease of $17.4 \%$ [48]. In 2016 ASCO gastrointestinal symposium, phase Ib KEYNOTE-028 study of pembrolizumab for PD-L1 positive advanced esophageal carcinoma reported that the ORR was $30.4 \%$ and PFS rate at 12 months was $21.7 \%$ [49]. Key trials of immune checkpoint inhibitors in patients with gastrointestinal cancer are summarized in table 1.

Table 1. Key trials of immune checkpoint inhibitors in patients with gastrointestinal cancer.

\begin{tabular}{|c|c|c|c|c|c|c|c|c|c|c|}
\hline Ref. no & Cancer type & Subtype & Drug & Phase & Line & $\mathrm{N}$ & ORR & DoR & PFS & OS \\
\hline \multirow[t]{2}{*}{37} & Colorectal cancer & MMR-deficient & Pembrolizumab & II & $>2$ & 10 & $40 \%$ & Not reached & $78 \%$ at 20 weeks & Not reached \\
\hline & & MMR-proficient & Pembrolizumab & II & $>2$ & 18 & $0 \%$ & Not applicable & $11 \%$ at 20 weeks & $\begin{array}{l}5.0 \text { months } \\
\text { (median) }\end{array}$ \\
\hline 38 & Colorectal cancer & PD-L1 positive & Pembrolizumab & $\mathrm{Ib}$ & $\geq 2$ & 23 & $4 \%$ & Ongoing & Ongoing & Ongoing \\
\hline 43 & Gastric cancer & PD-L1 positive & Pembrolizumab & $\mathrm{Ib}$ & $\geq 1$ & 39 & $22 \%$ & 40 weeks (median) & $26 \%$ at 6 months & $42 \%$ at 12 months \\
\hline 44 & Gastric cancer & $\begin{array}{l}\text { Irrespective of } \\
\text { PD-L1 status }\end{array}$ & Nivolumab & $\mathrm{I} / \mathrm{II}$ & $\geq 2$ & 59 & $12 \%$ & 7.1months (median) & Not mentioned & $38 \%$ at 12 months \\
\hline 48 & $\begin{array}{l}\text { Biliary tract } \\
\text { cancer }\end{array}$ & PD-L1 positive & Pembrolizumab & $\mathrm{Ib}$ & $>2$ & 24 & $17 \%$ & Ongoing & Ongoing & Ongoing \\
\hline 49 & $\begin{array}{l}\text { Esophageal } \\
\text { cancer }\end{array}$ & PD-L1 positive & Pembrolizumab & $\mathrm{Ib}$ & $>2$ & 23 & $30 \%$ & 40 weeks (median) & $30 \%$ at 6 months & Ongoing \\
\hline
\end{tabular}

DoR, duration of response; MMR, mismatch repair; ORR, overall response rate; OS, overall survival; PD-L1, programmed death ligand-1; PFS, progression-free survival. 


\section{Future perspective}

Current approaches in anticancer immunotherapy are shifting to multimodality and combination strategies. Multimodality strategy implies combining immunotherapy with another immunotherapeutics or other type of treatment such as conventional cytotoxic chemotherapy, radiotherapy, and $\mathrm{mAbs}$ directed to various growth factor receptors and their ligands. Conventional cytotoxic chemotherapy or radiotherapy usually destroy tumor cells, and released intracellular peptides, RNA, proteins can become useful tumor associated antigens. Lesterhuis et al. concurrently immunized patients with CRC who were receiving adjuvant oxaliplatin and capecitabine with keyhole limpet haemocyanin/CEA-pulsed DCs and successfully drew CEA-specific T-cell responses [50]. As suggested in advanced melanoma [51,52], PD-1/PD-L1 blockade may become an attractive combination partner with anti-CTLA-4 therapy. Trials combining immune checkpoint inhibitors with immunostimulatory mAbs, such as CD137-specific mAbs, are under investigation. Based on immunomodulating properties of vascular endothelial growth factor to impede infiltration of lymphocytes and DCs into tumor, ipilimumab has been combined with bevacizumab for patient with metastatic melanoma [53]. Intratumoral blood vessel changes in tumor specimens were more obvious in patients treated with bevacizumab plus ipilimumab, compared to those receiving ipilimumab alone.

In addition, anticancer vaccination can also be studied in combination with immune checkpoint blockade. Preliminary results of a trial with monocyte-derived DCs combined with PD-L1 checkpoint blockade for patients with pretreated advanced pancreatic cancer were presented in 2016 ASCO gastrointestinal symposium: median OS was reported to reach 18 months after primary diagnosis [54].

\section{Conclusion}

Cancer immunotherapy has shown promising results in various types of cancers. Especially immune checkpoint inhibitors are leading a recent renaissance of immune-mediated anticancer treatments. Early studies have suggested that immune checkpoint inhibition might also be effective in some patients with gastrointestinal cancers. However, the era of cancer immunotherapy in gastrointestinal cancers is in an inchoate stage. The immune system and immunosuppressive mechanisms surrounding GC or CRC are still obscure compared to malignant melanoma, renal cell carcinoma, or NSCLC. To increase efficacy of cancer immunotherapy in gastrointestinal cancers, we need to build more profound understanding of tumor immune system. Knowledge of potential relationships between tumor cells and their microenvironment is also essential in gastrointestinal malignancies.

\section{Competing Interests}

The authors have declared that no competing interest exists.

\section{References}

1. Ferlay J, Soerjomataram I, Dikshit R, Eser S, Mathers C, Rebelo M, et al. Cancer incidence and mortality worldwide: Sources, methods and major patterns in GLOBOCAN2012. Int J Cancer. 2015;136:E359-86.

2. Jung KW, Won YJ, Kong HJ, Oh CM, Lee DH, Lee JS. Prediction of cancer incidence and mortality in Korea, 2014. Cancer Res Treat. 2014;46:124-30.

3. Slankard-Chahinian M, Holland JF, Gordon RE, Becker J, Ohnuma T. Adoptive autoimmunotherapy. Cytotoxic effect of an autologous long-term T-cell line on malignant melanoma. Cancer. 1984;53:1066-72.

4. Parkinson DR, Abrams JS, Wiernik PH, Rayner AA, Margolin KA, Van Echo $\mathrm{DA}$, et al. Interleukin-2 therapy in patients with metastatic malignant melanoma: a phase II study. J Clin Oncol. 1990;8:1650-6.

5. Flaherty LE, Atkins M, Sosman J, Weiss G, Clark JI, Margolin K, et al. Outpatient biochemotherapy with interleukin-2 and interferon alfa-2b in patients with metastatic malignant melanoma: results of two phase II cytokine working group trials. J Clin Oncol. 2001;19:3194-202.

6. Bar MH, Sznol M, Atkins MB, Ciobanu N, Micetich KC, Boldt DH, et al. Metastatic malignant melanoma treated with combined bolus and continuous infusion interleukin-2 and lymphokine-activated killer cells. J Clin Oncol. 1990;8:1138-47.

7. Starr P. Encouraging results for pembrolizumab in head and neck cancer. Am Health Drug Benefits. 2015;8:16.

8. Prall F, Dührkop T, Weirich V, Ostwald C, Lenz P, Nizze H, Barten M. Prognostic role of CD8+ tumor-infiltrating lymphocytes in stage III colorectal cancer with and without microsatellite instability. Hum Pathol. 2004;35:808-16.

9. Karlsson M, Marits P, Dahl K, Dagöö T, Enerbäck S, Thörn M, Winqvist O. Pilot study of sentinel-node-based adoptive immunotherapy in advanced colorectal cancer. Ann Surg Oncol. 2010;17:1747-57.

10. Jiang J, Xu N, Wu C, Deng H, Lu M, Li M, et al. Treatment of advanced gastric cancer by chemotherapy combined with autologous cytokine-induced killer cells. Anticancer Res. 2006;26:2237-42.

11. Kim JH, Lee Y, Bae Y-S, Kim WS, Kim K, Im HY, et al. Phase I/II study of immunotherapy using autologous tumor lysate-pulsed dendritic cells in patients with metastatic renal cell carcinoma. Clin Immunol. 2007;125:257-67.

12. Testori A, Richards J, Whitman E, Mann GB, Lutzky J, Camacho L, et al. Phase III comparison of vitespen, an autologous tumor-derived heat shock protein gp96 peptide complex vaccine, with physician's choice of treatment for stage IV melanoma: The C-100-21 Study Group. J Clin Oncol. 2008;26:955-62.

13. Um S-J, Choi YJ, Shin H-J, Son CH, Park Y-S, Roh MS, et al. Phase I study of autologous dendritic cell tumor vaccine in patients with non-small cell lung cancer. Lung Cancer. 2010;70:188-94.

14. Hanna Jr. MG, Hoover Jr. HC, Vermorken JB, Harris JE, Pinedo HM. Adjuvant active specific immunotherapy of stage II and stage III colon cancer with an autologous tumor cell vaccine: first randomized phase III trials show promise. Vaccine. 2001;19:2576-82.

15. Conry RM, Curiel DT, Strong TV, Moore SE, Allen KO, Barlow DL, et al. Safety and immunogenicity of a DNA vaccine encoding carcinoembryonic antigen and hepatitis B surface antigen in colorectal carcinoma patients. Clin Cancer Res. 2002;8:2782-7.

16. Mazzaferro V, Coppa J, Carrabba MG, Rivoltini L, Schiavo M, Regalia E, et al. Vaccination with autologous tumor-derived heat-shock protein Gp96 after liver resection for metastatic colorectal cancer. Clin Cancer Res. 2003;9:3235-45.

17. Lasky JL, Panosyan EH, Plant A, Davidson T, Yong WH, Prins RM, et al. Autologous tumor lysate-pulsed dendritic cell immunotherapy for pediatric patients with newly diagnosed or recurrent high-grade gliomas. Anticancer Res. 2013;33:2047-56.

18. Kantoff PW, Higano CS, Shore ND, Berger ER, Small EJ, Penson DF, et al. Sipuleucel-T immunotherapy for castration-resistant prostate cancer. N Engl J Med. 2010;363:411-22.

19. Sadanaga N, Nagashima $H$, Mashino $K$, Tahara $K$, Yamaguchi $H$, Ohta $M$, et al. Dendritic cell vaccination with MAGE peptide is a novel therapeutic approach for gastrointestinal carcinomas. Clin Cancer Res. 2001;7:2277-84.

20. Kono K, Takahashi A, Sugai H, Fujii H, Choudhury AR, Kiessling R, et al. Dendritic cells pulsed with HER-2/neu-derived peptides can induce specific 
T-cell responses in patients with gastric cancer. Clin Cancer Res. 2002;8:3394-400.

21. Czerniecki BJ, Koski GK, Koldovsky U, Xu S, Cohen PA, Mick R, et al. Targeting HER-2/neu in early breast cancer development using dendritic cells with staged interleukin-12 burst secretion. Cancer Res. 2007;67:1842-52.

22. Kim JH, Kang TH, Noh KH, Kim S-H, Lee Y-H, Kim KW, et al. Enhancement of DC vaccine potency by activating the PI3K/AKT pathway with a small interfering RNA targeting PTEN. Immunol Lett. 2010;134:47-54.

23. Ojima T, Iwahashi M, Nakamura M, Matsuda K, Nakamori M, Ueda K, et al. Benefits of gene transduction of granulocyte macrophage colony-stimulating factor in cancer vaccine using genetically modified dendritic cells. Int J Oncol. 2007;31:931-9.

24. Morse MA, Niedzwiecki D, Marshall JL, Garrett C, Chang DZ, Aklilu M, et al. A randomized phase II study of immunization with dendritic cells modified with poxvectors encoding CEA and MUC1 compared with the same poxvectors plus GM-CSF for resected metastatic colorectal cancer. Ann Surg. 2016;258:879-86.

25. Barth RJ, Fischer DA, Wallace PK, Channon JY, Noelle RJ, Gui J, Ernstoff MS. A randomized trial of ex vivo CD40L activation of a dendritic cell vaccine in colorectal cancer patients: tumor-specific immune responses are associated with improved survival. Clin Cancer Res. 2010;16:5548-56.

26. Zhang L, Zhu W, Li J, Yang X, Ren Y, Niu J, Pang Y. Clinical outcome of immunotherapy with dendritic cell vaccine and cytokine-induced killer cell therapy in hepatobiliary and pancreatic cancer. Mol Clin Oncol. 2016;4:129-33.

27. Robert C, Thomas L, Bondarenko I, O'Day S, Weber J, Garbe C, et al. Ipilimumab plus dacarbazine for previously untreated metastatic melanoma. N Engl J Med. 2011;364:2517-26.

28. O'Mahony D, Morris JC, Quinn C, Gao W, Wilson WH, Gause B, et al. A pilot study of CTLA-4 blockade after cancer vaccine failure in patients with advanced malignancy. Clin Cancer Res. 2007;13:958-64.

29. Chung KY, Gore I, Fong L, Venook A, Beck SB, Dorazio P, et al. Phase II study of the anti-cytotoxic T-lymphocyte-associated antigen 4 monoclonal antibody, tremelimumab, in patients with refractory metastatic colorectal cancer. J Clin Oncol. 2010;28:3485-90.

30. Ralph C, Elkord E, Burt DJ, O'Dwyer JF, Austin EB, Stern PL, et al. Modulation of lymphocyte regulation for cancer therapy: a phase II trial of tremelimumab in advanced gastric and esophageal adenocarcinoma. Clin Cancer Res. 2010:16:1662-72.

31. Barber DL, Wherry EJ, Masopust D, Zhu B, Allison JP, Sharpe AH, et al. Restoring function in exhausted CD8 T cells during chronic viral infection. Nature. 2006:439:682-7.

32. Robert C, Schachter I, Long GV, Arance A, Grob JJ, Mortier L, et al Pembrolizumab versus ipilimumab in advanced melanoma. N Engl J Med. 2015;372:2521-32.

33. Herbst RS, Baas P, Kim DW, Felip E, Pérez-Gracia JL, Han JY, et al. Pembrolizumab versus docetaxel for previously treated, PD-L1-positive, advanced non-small-cell lung cancer (KEYNOTE-010): a randomised controlled trial. Lancet. 2016;387:1540-50.

34. Brahmer J, Reckamp KL, Baas P, Crinò L, Eberhardt WEE, Poddubskaya E, et al. Nivolumab versus docetaxel in advanced squamous-cell non-small cell lung cancer. N Engl J Med. 2015;373:123-35.

35. Martin-Liberal J, Kordbacheh T, Larkin J. Safety of pembrolizumab for the treatment of melanoma. Expert Opin Drug Saf. 2015;14:957-64.

36. Llosa NJ, Cruise M, Tam A, Wicks EC, Hechenbleikner EM, Taube JM, et al. The vigorous immune microenvironment of microsatellite instable colon cancer is balanced by multiple counter-inhibitory checkpoints. Cancer Discov. 2015;5:43-51.

37. Le DT, Uram JN, Wang H, Bartlett BR, Kemberling H, Eyring AD, et al. PD-1 blockade in tumors with mismatch repair deficiency. $\mathrm{N}$ Engl J Med. 2015;372:2509-20.

38. O'Neil BH, Wallmark J, Lorente D, Elez E, Raimbourg J, Gomez-Roca C, et al. Pembrolizumab (MK-3475) for patients with advanced colorectal carcinoma (CRC): Preliminary results from KEYNOTE-028. Eur J Cancer. 2015;51 (suppl 3): abstr502.

39. Bustin SA, Li S-R, Phillips S, Dorudi S. Expression of HLA Class II in colorectal cancer: evidence for enhanced immunogenicity of microsatellite-instability-positive tumours. Tumor Biol. 2001;22:294-8.

40. Bauer K, Michel S, Reuschenbach M, Nelius N, Doeberitz M von K, Kloor M. Dendritic cell and macrophage infiltration in microsatellite-unstable and microsatellite-stable colorectal cancer. Fam Cancer. 2011;10:557-65.

41. Correale P, Rotundo MS, Del Vecchio MT, Remondo C, Migali C, Ginanneschi $\mathrm{C}$, et al. Regulatory (FoxP3+) T-cell tumor infiltration is a favorable prognostic factor in advanced colon cancer patients undergoing chemo or chemoimmunotherapy. J Immunother. 2010;33:435-41.

42. Ling A, Edin S, Wikberg ML, Öberg $\AA$, Palmqvist R. The intratumoural subsite and relation of CD8+ and FOXP3+ T lymphocytes in colorectal cancer provide important prognostic clues. Br J Cancer. 2014;110:2551-9.

43. Muro K, Bang Y, Shankaran V, Geva R, Catenacci DVT, Gupta S, et al. Relationship between PD-L1 expression and clinical outcomes in patients with advanced gastric cancer treated with the anti-PD-1 monoclonal antibody pembrolizumab (Pembro; MK-3475) in KEYNOTE-012. J Clin Oncol. 2015; 33 (suppl 3; abstr 3).

44. Le DT, Bendell JC, Calvo E, Kim JW, Ascierto PA, Sharma P, et al. Safety and activity of nivolumab monotherapy in advanced and metastatic $(\mathrm{A} / \mathrm{M})$ gastric or gastroesophageal junction cancer (GC/GEC): results from the CheckMate-032 study. J Clin Oncol 2016;34 (suppl 4S): abstr6.

45. Rizvi NA, Hellmann MD, Snyder A, Kvistborg P, Makarov V, Havel JJ, et al. Cancer immunology. Mutational landscape determines sensitivity to PD-1 blockade in non-small cell lung cancer. Science. 2015;348:124-8.

46. Calles A, Liao X, Sholl LM, Rodig SJ, Freeman GJ, Butaney M, et al. Expression of PD-1 and its ligands, PD-L1 and PD-L2, in smokers and never smokers with KRAS-mutant lung cancer. J Thorac Oncol. 2015;10:1726-35.

47. Nakamura H, Arai Y, Totoki Y, Shirota T, Elzawahry A, Kato M, et al. Genomic spectra of biliary tract cancer. Nat Genet. 2015;47:1003-10.

48. Bang YJ, De Braud F, Piha-Paul S, Hollebecque A, Abdul Razak AR, et al. Safety and efficacy of pembrolizumab (MK-3475) in patients (pts) with advanced biliary tract cancer: Interim results of KEYNOTE-028. Eur J Cancer. 2106;51 (suppl 3; abstr 525).

49. Doi T, Piha-Paul SA, Jalal SI, Mai-Dang H, Saraf S, Koshiji M, et al. Updated results for the advanced esophageal carcinoma cohort of the phase $\mathrm{Ib}$ KEYNOTE-028 study of pembrolizumab (MK-3475). J Clin Oncol 2016;34 (suppl 4S; abstr 7)

50. Lesterhuis WJ, de Vries IJM, Aarntzen EA, de Boer A, Scharenborg NM, van de Rakt $\mathrm{M}$, et al. A pilot study on the immunogenicity of dendritic cell vaccination during adjuvant oxaliplatin/capecitabine chemotherapy in colon cancer patients. Br J Cancer. 2010;103:1415-21.

51. Larkin J, Chiarion-Sileni V, Gonzalez R, Grob JJ, Cowey CL, Lao CD, et al. Combined nivolumab and ipilimumab or monotherapy in untreated melanoma. N Engl J Med. 2015;373:23-34.

52. Postow MA, Chesney J, Pavlick AC, Robert C, Grossmann K, McDermott D, et al. Nivolumab and ipilimumab versus ipilimumab in untreated melanoma. $\mathrm{N}$ Engl J Med. 2015;372:2006-17.

53. Hodi FS, Lawrence D, Lezcano C, Wu X, Zhou J, Sasada T, et al. Bevacizumab plus ipilimumab in patients with metastatic melanoma. Cancer Immunol Res. 2014;2:632-42.

54. Nesselhut J, Marx D, Cillien N, Lange H, Regalo G, Herrmann M, et al. Dendritic cells generated with PDL-1 checkpoint blockade for treatment of advanced pancreatic cancer. J Clin Oncol 2015;33 (suppl 3; abstr 4128). 